\title{
A Note on the Minimal Period Problem for Second Order Hamiltonian Systems
}

\author{
Huafeng Xiao ${ }^{1,2}$ \\ ${ }^{1}$ School of Mathematics and Information Science, Guangzhou University, Guangzhou 510006, China \\ ${ }^{2}$ Key Laboratory of Mathematics and Interdisciplinary Science of Guangdong, Higher Education Institutes, \\ Guangzhou University, Guangzhou 510006, China
}

Correspondence should be addressed to Huafeng Xiao; huafeng@gzhu.edu.cn

Received 26 April 2014; Revised 21 July 2014; Accepted 19 August 2014; Published 20 October 2014

Academic Editor: Anna Capietto

Copyright (C) 2014 Huafeng Xiao. This is an open access article distributed under the Creative Commons Attribution License, which permits unrestricted use, distribution, and reproduction in any medium, provided the original work is properly cited.

We study periodic solutions of second order Hamiltonian systems with even potential. By making use of generalized Nehari manifold, some sufficient conditions are obtained to guarantee the multiplicity and minimality of periodic solutions for second order Hamiltonian systems. Our results generalize the outcome in the literature.

\section{Introduction}

Denote by $\mathbb{N}, \mathbb{Z}, \mathbb{R}^{*}, \mathbb{R}$ the sets of all natural numbers, integers, nonnegative real numbers, and real numbers, respectively. For $N \in \mathbb{N}$, denote by $\mathbb{R}^{N}$ the $N$-dimensional Euclidean space with the usual inner product $(\cdot, \cdot)$ and norm $|\cdot|$.

Consider the second order Hamiltonian systems

$$
\ddot{x}+A x+V^{\prime}(x)=0, \quad \forall x \in \mathbb{R}^{N} .
$$

Assume that

(A1) $A$ is a symmetric, negative semidefinite matrix;

(V1) $V \in C^{1}\left(\mathbb{R}^{N}, \mathbb{R}\right)$ and $V(x) \geq 0$ for all $x \in \mathbb{R}^{N}$;

(V2) $V(x)=o\left(|x|^{2}\right)$ as $x \rightarrow 0$;

(V3) $|x|^{-2} V(x) \rightarrow+\infty$ as $|x| \rightarrow \infty$;

(V4) for any $x \in \mathbb{R}^{N}$ with $|x|=1, \lambda \rightarrow(1 / \lambda)\left(V^{\prime}(\lambda x), x\right)$ is strictly increasing on $(0, \infty)$;

(V5) there exist $s>2$ and $a_{1}, a_{2}>0$ such that $|V(x)| \leq$ $a_{1}+a_{2}|x|^{s}$

(V6) $V$ is even; that is, $V(-x)=V(x)$ for all $x \in \mathbb{R}^{N}$.

When $A=0$, in his pioneering work [1] of 1978, Rabinowitz established the existence of periodic solutions of (1) when $V$ satisfies (V1), (V2), and the well-known ARcondition:

$$
\begin{array}{r}
\exists \mu>2, \quad r_{0}>0 \text { such that } 0<\mu V(x) \leq\left(x, V^{\prime}(x)\right), \\
\forall|x| \geq r_{0} .
\end{array}
$$

He conjectured that (1) possesses a nonconstant solution with any prescribed minimal period under the same assumptions. Since then, many authors devoted themselves to the study of periodic solutions with prescribed minimal period of (1).

When $A=0$, in 1993, Long ( $c f .[2])$ proved the existence of $T$-periodic solutions with minimal period $T$ or $T / 3$ of (1) under the assumptions that $V \in C^{2}\left(\mathbb{R}^{N}, \mathbb{R}^{*}\right)$ satisfies (V2), (V6), and (V7). Releasing assumption (V6), Long showed that (1) possesses periodic solutions with minimal periodic $T / k$ for some integer $k$ satisfying $1 \leq k \leq n+2$ (cf. [3]) or $1 \leq k \leq n+1$ (cf. [4]). In 2001, Fei et al. (cf. [5]) proved that (1) has a nonconstant periodic solution with any prescribed minimal period when $V \in C^{2}\left(\mathbb{R}^{N}, \mathbb{R}^{*}\right)$ satisfies (V2), (V6), and $(\mathrm{V} 7)$, and $V^{\prime \prime}(x)$ is positive semidefinite.

When $A$ is positive semidefinite, $V \in C^{2}\left(\mathbb{R}^{N}, \mathbb{R}^{*}\right)$ satisfies (V2) and (V7); in 1997, Fei and Wang (cf. [6]) showed that (1) possesses nonconstant $T$-periodic solutions with minimal periodic $T / k$ for some odd interger $k$ satisfying $1 \leq k \leq$ 
$2\left(i_{T}(A)+v_{T}(A)\right)+3$, where $i_{T}(A)=\sum_{m=0}^{\infty} M^{-}\left(T_{m}(A)\right)$, $v_{T}(A)=\sum_{m=0}^{\infty} M^{0}\left(T_{m}(A)\right)$, and $T_{m}(A)=(2 \pi m / T)^{2} I-A$. If $V$ is even, replacing (V7) by (V3), and the following condition

$\exists \beta \in[0,2]$,

$d \geq 0$ such that $\mu V(x)-\left(V^{\prime}(x), x\right) \leq d|x|^{\beta}, \quad \forall|x| \geq r_{0}$,

Fei and Wang ( $c f$. [7]) estimated that the minimal period is not smaller than $T /\left(i_{T}(A)+v_{T}(A)+2\right)$.

The above results on minimal period problem were obtained by making use of index theory. Another method being used to study such a problem is Nehari manifold. As is well known, the Nehari manifold, introduced by Nehari (cf. $[8,9])$, has been used widely to study the existence of ground state solutions of partial differential equations ( $c f$. $[10-14])$ and that of (1) (cf. $[13,15,16])$. A ground state solution of a system is a solution which possesses the minimal energy of all solutions of the system. However, such kind of solutions may not have prescribed minimal periods. In 1981, Ambrosetti and Mancini made use of Nehari manifold to study the existence of periodic solutions with any prescribed minimal period of first order Hamiltonian systems with convex potential (cf. [17]). In 2010, Xiao (cf. [18]) proved that (1) possesses a periodic solution with any prescribed minimal period and when $A=0, V$ satisfies (V6) and the following assumption:

(V8)

$$
\begin{array}{r}
V \in C^{2}\left(\mathbb{R}^{N}, \mathbb{R}\right), \quad 0<v\left(V^{\prime}(x), x\right) \leq\left(V^{\prime \prime}(x) x, x\right), \\
\forall x \in \mathbb{R}^{N} \backslash\{0\},
\end{array}
$$

where $v>1$. For more results on this direction, we refer to $[19,20]$.

Motivated by $[13,17,18]$, in this paper, we consider the multiplicity and minimality of periodic solutions of (1) under the assumption (A1), (V1)-(V6). Our main result reads as follows.

Theorem 1. Assume that (A1), (V1)-(V6) hold. Then, for any $T>0$, (1) possesses a periodic solution with minimal period $T$.

Corollary 2. If $A=0$ and $V$ satisfies (V6) and (V8), then, for any $T>0$, (1) possesses a periodic solution with minimal period $T$.

Theorem 3. Assume that (A1), (V1)-(V6) hold. Then, for any $T>0$, (1) possesses infinitely many pairs of T-periodic solutions.

The rest of this paper is divided into two parts. In Section 2, we establish the variational functional and state some useful lemmas. In Section 3, we introduce Nehari Manifold and prove our main results.

\section{Preliminary}

Denote by $S_{T}=\mathbb{R} /(T \mathbb{Z}) . H=W^{1,2}\left(S_{T}, \mathbb{R}^{N}\right)$ is a Hilbert space equipping with the usual norm and inner product

$$
\begin{aligned}
\|x\|_{H}^{2} & =\int_{0}^{T}\left(|\dot{x}|^{2}+|x|^{2}\right) d t, \\
\langle x, y\rangle_{H} & =\int_{0}^{T}[(\dot{x}, \dot{y})+(x, y)] d t .
\end{aligned}
$$

The variational functional defined on $H$, corresponding to (1), is

$$
\begin{aligned}
\varphi(x)=\int_{0}^{T}[ & \frac{1}{2}|\dot{x}(t)|^{2} \\
& \left.-\frac{1}{2}(A x(t), x(t))-V(x(t))\right] d t .
\end{aligned}
$$

If (A1), (V1)-(V5) hold, then $\varphi$ is continuous differentiable on $H$ and

$$
\begin{gathered}
\left\langle\varphi^{\prime}(x), y\right\rangle_{H}=\int_{0}^{T}[(\dot{x}(t), \dot{y}(t))-(A x(t), y(t)) \\
\left.-\left(V^{\prime}(x(t)), y(t)\right)\right] d t, \\
\forall x, y \in H .
\end{gathered}
$$

Moreover, $\phi(x)=\int_{0}^{T} V(x(t)) d t$ is weakly continuous and $\phi^{\prime}$ : $H \rightarrow H^{*}$ is compact.

Define a subspace of $H$ as follows:

$$
E=\{x \in H \mid x(-t)=-x(t)\} .
$$

Then $E$ is a closed subspace of $H$. If $x \in E$, it has a Fourier expansion

$$
x(t)=\sum_{n=1}^{\infty} a_{n} \sin \frac{2 \pi n t}{T} .
$$

Obviously, $E \cap \mathbb{R}^{N}=\{0\}$. We can define an equivalent inner product on $E$

$$
\langle x, y\rangle_{1}=\int_{0}^{T}(\dot{x}(t), \dot{y}(t)) d t .
$$

Let us state a useful lemma and omit the proof. One can find the details in [21].

Lemma 4. If $x$ is a critical point of $\varphi$ on $E$, then $x$ is a critical point of $\varphi$ on $H$.

Define an operator on $E$ by extending bilinear form

$$
\langle L x, y\rangle_{1}=\int_{0}^{T}[(\dot{x}(t), \dot{y}(t))-(A x(t), y(t))] d t .
$$

It is easy to verify that $L$ is a linear self-adjoint operator. Since $A$ is a negative semidefinite matrix, then $L$ has a sequence of eigenvalues

$$
0<\lambda_{1} \leq \lambda_{2} \leq \cdots \leq \lambda_{n} \leq \cdots
$$


with $\lambda_{n} \rightarrow+\infty$ as $n \rightarrow \infty$. Thus for any $x \in E \backslash\{0\}$, $\langle L x, x\rangle_{1} \geq \lambda_{1}\|x\|_{1}^{2}$, where $\|\cdot\|_{1}$ denotes the norm induced by $\langle\cdot, \cdot\rangle_{1}$. Define another equivalent inner product on $E$

$$
\langle x, y\rangle=\langle L x, y\rangle_{1} .
$$

Then, for all $x \in E$, there exist $C_{1}, C_{2}>0$ such that $C_{1}\|x\| \leq$ $\|x\|_{1} \leq C_{2}\|x\|$, where $\|\cdot\|$ denotes the norm induced by $\langle\cdot, \cdot\rangle$.

Functional (6) can be rewritten as

$$
\varphi(x)=\frac{1}{2}\|x\|^{2}-\int_{0}^{T} V(x(t)) d t .
$$

In the end of this section, we introduce two useful lemmas.

Lemma 5 (see [22]). If $x \in H$ and $\int_{0}^{T} x(t) d t=0$, then

$$
\begin{aligned}
\int_{0}^{T}|x(t)|^{2} d t & \leq \frac{T^{2}}{4 \pi^{2}} \int_{0}^{T}|\dot{x}(t)|^{2} d t, \\
\|x\|_{\infty}^{2} & \leq \frac{T}{12} \int_{0}^{T}|\dot{x}|^{2} d t,
\end{aligned}
$$

where $\|\cdot\|_{\infty}=\max _{t \in[0, T]}|x(t)|$.

If $x \in E, \int_{0}^{T} x(t) d t=0$. Thus elements of $E$ satisfy the above two inequalities.

Let $X$ be a Banach space such that the unit sphere $S^{1}$ in $X$ is a submanifold of class (at least) $C^{1}$ and let $J \in C^{1}\left(S^{1}, \mathbb{R}\right)$. A sequence $\left\{x_{n}\right\} \subset S^{1}$ is called (PS) sequence for $J$ if it satisfies $\left\{J\left(x_{n}\right)\right\}$ is bounded and $J^{\prime}\left(x_{n}\right) \rightarrow 0$ as $n \rightarrow \infty$. We say that $J$ satisfies the $(P S)$ condition if every $(P S)$ sequence contains a convergent subsequence.

Lemma 6 (see [13]). If $X$ is infinite-dimensional and $J \in$ $C^{1}\left(S^{1}, \mathbb{R}\right)$ is bounded below and satisfies the $(P S)$ condition on $S^{1}$, then J has infinitely many pairs of critical points.

\section{Proofs of the Main Results}

Denote by $h(x)=\left\langle\varphi^{\prime}(x), x\right\rangle$. We define the Nehari manifold

$$
\mathscr{M}=\{x \in E \backslash\{0\} \mid h(x)=0\} .
$$

Denote by $S=\{x \in E \mid\|x\|=1\}$. Fixing $x \in S$, define $g_{x}(r)=\varphi(r x)$ for $r \in(0, \infty)$. On the one hand, if $x \in \mathcal{M}$, then $y=x /\|x\| \in S$ and

$$
\begin{aligned}
g_{y}^{\prime}(\|x\|) & =\left\langle\varphi^{\prime}(\|x\| y), y\right\rangle \\
& =\frac{\left\langle\varphi^{\prime}(x), x\right\rangle}{\|x\|}=\frac{h(x)}{\|x\|}=0 .
\end{aligned}
$$

On the other hand, if $g_{x}^{\prime}(r)=0$, then

$$
\begin{aligned}
h(r x) & =\left\langle\varphi^{\prime}(r x), r x\right\rangle=r\left\langle\varphi^{\prime}(r x), x\right\rangle \\
& =r g_{x}^{\prime}(r)=0 .
\end{aligned}
$$

Thus, for any $x \in S, g_{x}^{\prime}(r)=0$ if and only if $r x \in \mathscr{M}$. The following lemma shows that $\mathscr{M} \neq \emptyset$.
Lemma 7. For any $x \in S$, there exists a unique $r_{0}$ dependent on $x$ such that

$$
g_{x}\left(r_{0}\right)=\sup _{r \in \mathbb{R}^{*}} g_{x}(r) .
$$

Proof. By the definition of $g_{x}$, we have

$$
g_{x}(r)=\varphi(r x)=\frac{1}{2} r^{2}-\int_{0}^{T} V(r x(t)) d t .
$$

By (V2), for any $\epsilon>0$, there exists $\delta_{\epsilon}>0$ such that $V(x) \leq$ $\epsilon|x|^{2}$ for $|x| \leq \delta_{\epsilon}$. If $x \in S$, then Lemma 5 implies that $\|x\|_{L^{\infty}} \leq$ $\sqrt{T / 12} C_{2}$ and $|x(t)| \leq \sqrt{T / 12} C_{2}$ for $t \in[0, T]$. Fixing $\epsilon_{1}<$ $3 /\left(T^{2} C_{2}^{2}\right)$, there exists $r_{1}=\delta_{\epsilon_{1}} /\left(\sqrt{T / 12} C_{2}\right)$ such that for all $x \in S$ and $0<r<r_{1}$

$$
\begin{aligned}
\varphi(r x) & =\frac{1}{2} r^{2}-\int_{0}^{T} V(r x(t)) d t \\
& \geq \frac{1}{2} r^{2}-\epsilon_{1} r^{2} \int_{0}^{T}|x|^{2} d t>\frac{r^{2}}{4} .
\end{aligned}
$$
denote

Let $\alpha_{0}=1 / T \int_{0}^{T}|x(t)| d t$. Then $\alpha_{0}>0$. For any $0<\alpha<\alpha_{0}$,

$$
A_{\alpha}=\{t \in[0, T]|| x(t) \mid \geq \alpha\} .
$$

Then meas $\left(A_{\alpha}\right)>0$, where meas $\left(A_{\alpha}\right)$ denotes the measure of $A_{\alpha}$. Thus (V1) and (V3) yield that

$$
\begin{aligned}
\frac{g_{x}(r)}{r^{2}} & =\frac{1}{2}-\int_{0}^{T} \frac{V(r x(t))}{r^{2}} d t \\
& \leq \frac{1}{2}-\int_{A_{\alpha}} \frac{V(r x(t))}{|r x(t)|^{2}}|x(t)|^{2} d t \longrightarrow-\infty,
\end{aligned}
$$

$$
\text { as } r \longrightarrow \infty \text {. }
$$

Consequently, there exists $r_{2}>0$ dependent on $x$ such that $g_{x}(r)<0$ for $r \geq r_{2}$. By the mean value theorem, there exists $r_{0}>0$ dependent on $x$ such that $g_{x}\left(r_{0}\right)=\sup _{r \in \mathbb{R}^{*}} g_{x}(r)$ and $g_{x}^{\prime}\left(r_{0}\right)=0$.

By the definition, $g_{x}$ is continuous differentiable on $(0,+\infty)$. Differentiating $g_{x}$, we have

$$
\begin{aligned}
\frac{g_{x}^{\prime}(r)}{r} & =1-\frac{1}{r} \int_{0}^{T}\left(V^{\prime}(r x), x\right) d t \\
& =1-\int_{0}^{T}|x|^{2}\left(\frac{1}{r|x|} V^{\prime}(r|x| y), y\right) d t,
\end{aligned}
$$

where $y=x /|x|$. Because of (V4), $g_{x}^{\prime}(r) / r$ is strictly decreasing on $(0,+\infty)$. Thus $g_{x}^{\prime}(r) / r$ and hence $g_{x}^{\prime}(r)$ has a unique zero point on $(0,+\infty)$. It follows that $g_{x}(r)$ has a unique critical point, which is a maximum.

Remark 8. Fixing $x \in E \backslash\{0\}$, then $x /\|x\| \in S$. Obviously, $\varphi(r x)=\varphi(r\|x\| \cdot x /\|x\|)$ and $\sup _{r \in \mathbb{R}^{*}} \varphi(r x)=$ $\sup _{r \in \mathbb{R}^{*}} \varphi(r x /\|x\|)$. If we extend the definition of $g_{x}$ to $E \backslash\{0\}$, then Lemma 7 implies that there exists a unique $r_{x}>0$ such that $g_{x}\left(r_{x}\right)=\sup _{r \in \mathbb{R}^{*}} \varphi(r x)$. Also, $g_{x}^{\prime}(r)>0$ for $0<r<r_{x}$ and $g_{x}^{\prime}(r)<0$ for $r>r_{x}$.

Now, we study the properties of $\varphi$ restricted on $\mathscr{M}$. 
Lemma 9. Consider the following:

$c_{0}=\inf _{x \in M} \varphi(x)>0$.

Proof. For any $x \in \mathscr{M}$, both Lemma 7 and Remark 8 imply that $\varphi(x)=\sup _{r \in \mathbb{R}^{*}} \varphi(r x)>r_{1}^{2} / 16$. Hence $c_{0}=\inf _{x \in M} \varphi(x) \geq$ $r_{1}^{2} / 16>0$.

Lemma 10. $\varphi$ satisfies the $(P S)$ condition on $\mathscr{M}$.

Proof. Assume that $\left\{x_{n}\right\} \subset \mathscr{M}$ is a (PS) sequence of $\varphi$. Then there exists $d \geq 0$ such that $\varphi\left(x_{n}\right) \leq d$ and $\varphi^{\prime}\left(x_{n}\right) \rightarrow 0$ as $n \rightarrow \infty$. We show that $\left\{x_{n}\right\}$ is bounded. Suppose, to the opposite, that $\left\|x_{n}\right\| \rightarrow \infty$ as $n \rightarrow \infty$. Set $y_{n}=x_{n} /\left\|x_{n}\right\|$. Then $\left\{y_{n}\right\} \subset S$. Passing to a subsequence, $y_{n} \rightarrow y_{0}$. If $y_{0}=0$, then $y_{n}(t) \Rightarrow 0$ uniformly for $t \in[0, T]$. Hence, for any $r>0$, we have

$$
\begin{aligned}
d \geq \varphi\left(x_{n}\right) & =\varphi\left(\left\|x_{n}\right\| y_{n}\right) \geq \varphi\left(r y_{n}\right) \\
& =\frac{1}{2} r^{2}-\int_{0}^{T} V\left(r y_{n}(t)\right) d t \longrightarrow \frac{1}{2} r^{2} .
\end{aligned}
$$

This is a contradiction if $r>\sqrt{2 d}$. So $y_{0} \neq 0$. Arguing similarly to [23], there exist $\delta_{1}>0, \delta_{2}>0$ such that

$$
\text { meas }\left(\left\{t \in[0, T]|| y_{0}(t) \mid \geq \delta_{1}\right\}\right) \geq \delta_{2} \text {. }
$$

Denote $A=\left\{t \in[0, T]|| y_{0}(t) \mid \geq \delta_{1}\right\}$. But (V3) implies that

$$
\begin{aligned}
0 \leq & \frac{\varphi\left(x_{n}\right)}{\left\|x_{n}\right\|^{2}} \leq \frac{1}{2} \\
& -\int_{A} \frac{V\left(\left\|x_{n}\right\| y_{n}\right)}{\left\|x_{n}\right\|^{2}\left|y_{n}\right|^{2}}\left|y_{n}\right|^{2} d t \longrightarrow-\infty, \quad \text { as } n \longrightarrow \infty .
\end{aligned}
$$

This is a contradiction. Hence $\left\{\left\|x_{n}\right\|\right\}$ is bounded. Since $\phi^{\prime}$ is compact, passing to a subsequence, $\left\{\phi^{\prime}\left(x_{n}\right)\right\}$ converges strongly. Since $x_{n}=\varphi^{\prime}\left(x_{n}\right)+\phi^{\prime}\left(x_{n}\right)$, then $\left\{x_{n}\right\}$ contains a convergent subsequence.

Define the maps as follows:

$$
\begin{aligned}
& \widehat{m}: E \backslash\{0\} \longrightarrow \mathscr{M}, \quad \widehat{m}(x)=r_{x} x, \\
& m=\left.\widehat{m}\right|_{S}: S \longrightarrow \mathscr{M}, \quad m=\left.m\right|_{S}, \\
& \widehat{\Psi}: E \backslash\{0\} \longrightarrow \mathbb{R}, \quad \widehat{\Psi}(x)=\varphi(\widehat{m}(x)), \\
& \Psi: S \longrightarrow \mathbb{R}, \quad \Psi=\left.\widehat{\Psi}\right|_{S},
\end{aligned}
$$

where $r_{x}$ is defined on Remark 8 .

Lemma 11. Assume that (V1)-(V4) hold. Then the following statements hold:

(B1) there exists a normalization function $\varphi$ such that

$$
u \longmapsto \psi(u):=\int_{0}^{\|u\|} \varphi(t) d t \in C^{1}(E \backslash\{0\}, \mathbb{R}) ;
$$

$J:=\psi^{\prime}$ is bounded on bounded sets and $J(w) w=1$ for all $w \in S$;
(B2) for each $w \in E \backslash\{0\}$ there exists $s_{w}$ such that if $\alpha_{w}(s):=$ $\Phi(s w)$, then $\alpha_{w}^{\prime}(s)>0$ for $0<s<s_{w}$ and $\alpha_{w}^{\prime}(s)<0$ for $s>s_{w}$;

(B3) there exists $\delta>0$ such that $s_{w} \geq \delta$ for all $w \in S$ and for each compact subset $W \subset S$ there exists a constant $C_{W}$ such that $s_{w} \leq C_{S}$ for all $w \in W$.

Proof. It follows from the discussion in [13] and Remark 8 that (B1), (B2) hold, and $r_{x} \geq r_{1} / 2$ for all $x \in S$. Next, we show that the last part of (B3) holds. Arguing by contradiction, we suppose that there exists a compact subset $W \in S$ and a sequence $\left\{r_{x_{n}} x_{n}\right\} \subset \mathscr{M}$, where $\left\{x_{n}\right\} \subset W$ and $\left\{r_{x_{n}}\right\} \subset \mathbb{R}^{*} \backslash\{0\}$, such that $r_{x_{n}} \rightarrow \infty$ as $n \rightarrow \infty$. Since $W$ is compact and $x_{n} \in W$, passing to a subsequence, $x_{n} \rightarrow x_{0} \in S$. Since the embedding of $H$ into $C\left(S_{T}, R^{N}\right)$ is compact and $x_{n} \in W$, then $x_{n}(t) \Rightarrow x_{0}(t)$ uniformly for $t \in[0, T]$. Denote by $A_{x_{0}}=\{t \in$ $\left.[0, T]|| x_{0}(t)\left|\geq 1 / T \int_{0}^{T}\right| x_{0}(t) \mid d t\right\}$. Since $r_{x_{n}}\left|x_{n}(t)\right| \rightarrow \infty$, if $t \in A_{x_{0}}$, then Fatou's lemma yields

$$
\begin{aligned}
& \frac{\phi\left(r_{x_{n}} x_{n}\right)}{r_{x_{n}}^{2}} \geq \int_{A_{x_{0}}} \frac{V\left(r_{x_{n}} x_{n}(t)\right)}{r_{x_{n}}^{2}\left|x_{n}(t)\right|^{2}}\left|x_{n}(t)\right|^{2} d t \rightarrow \infty, \\
& \text { as } n \longrightarrow \infty \text {. }
\end{aligned}
$$

Thus $\varphi\left(r_{x_{n}} x_{n}\right)=1 / 2 r_{x_{n}}^{2}-\phi\left(r_{x_{n}} x_{n}\right) \rightarrow-\infty$, which contradicts with the fact that $\varphi\left(r_{x_{n}} x_{n}\right)>r_{1}^{2} / 16>0$. Consequently, there exists $C_{W}>0$ such that $r_{x} \leq C_{W}$ for all $x \in W$ and (B3) holds.

Lemma 12 (see [13]). The mapping $\widehat{m}$ is continuous and $m$ is a homeomorphism between $S$ and $M$.

Lemma 13 (see [13]). Consider the following.

(1) $\widehat{\Psi}, \Psi \in C^{1}(S, \mathbb{R})$ and

$$
\begin{array}{r}
\left\langle\widehat{\Psi}^{\prime}(x), y\right\rangle=\frac{\|\widehat{m}(x)\|}{\|x\|}\left\langle\Psi^{\prime}(\widehat{m}(x)), y\right\rangle, \\
\forall x, y \in E, \quad x \neq 0, \\
\left\langle\Psi^{\prime}(x), y\right\rangle=\|m(x)\|\left\langle\Psi^{\prime}(m(x)), y\right\rangle, \\
\forall y \in T_{x}(S),
\end{array}
$$

where $T_{x}(S)=\{y \in E \mid\langle\varphi(x), y\rangle=0\}$ is the tangent space of $S$ at $x$.

(2) If $\left\{x_{n}\right\}$ is a (PS) sequence for $\Psi$, then $\left\{m\left(x_{n}\right)\right\}$ is a (PS) sequence for $\varphi$. If $\left\{x_{n}\right\} \subset \mathscr{M}$ is a bounded (PS) sequence for $\varphi$, then $\left\{m^{-1}\left(x_{n}\right)\right\}$ is a (PS) sequence for $\Psi$.

(3) $x$ is a critical point of $\Psi$ if and only if $m(x)$ is a nontrivial critical point of $\varphi$. Moreover, the corresponding values of $\Psi$ and $\varphi$ coincide and $\inf _{S} \Psi=\inf _{\mathscr{M}} \varphi$.

(4) If $\varphi$ is even, then so is $\Psi$.

Lemma 14. If $\varphi$ satisfies the (PS) condition, so does $\Psi$.

Proof. Assume that $\left\{x_{n}\right\}$ is a (PS) sequence for $\Psi$. According to Lemma 13, $\left\{m\left(x_{n}\right)\right\} \subset \mathscr{M}$ is a $(P S)$ sequence for $\varphi$. Since 
$\varphi$ satisfies the (PS) condition, passing to a subsequence, $m\left(x_{n}\right) \rightarrow y$. Thus $\left\{x_{n}\right\}$ contains a convergent subsequence, which converges to $m^{-1}(y)$. Hence $\Psi$ satisfies the (PS) condition.

Proof of Theorem 1. According to Lemmas 9 and 13, $\inf _{x \in S} \Psi(x)=\inf _{x \in \mathscr{M}} \varphi(x)=c_{0}$. Let $\left\{y_{n}\right\}$ be a minimizing sequence for $\Psi$ restricted to $S$. By Ekeland's variational principle, we may assume that $\Psi^{\prime}\left(y_{n}\right) \rightarrow 0$ as $n \rightarrow \infty$. The (PS) condition implies that $\left\{y_{n}\right\}$ contains a converging subsequence, whose limit is denoted by $y_{0}$. Thus $y_{0}$ is a critical point of $\Psi$. According to Lemma 13 again, $x_{0}=m\left(y_{0}\right)$ is a critical point of $\varphi$, which is also a nonconstant $T$-periodic solution of (1).

Claim. $x_{0}$ has minimal period $T$.

Suppose, to the opposite, that $x_{0}$ has minimal period $T / k$, where $k \geq 2$ is an integer. Let $y_{0}(t)=x_{0}(t / k)$. Obviously, $y_{0} \in E$. It follows from Lemma 7 that there exists $\bar{r}>0$ such that $\bar{r} y_{0} \in \mathscr{M}$. It follows that

$$
\begin{aligned}
\inf _{x \in \mathscr{M}} \varphi(x) & \leq \varphi\left(\bar{r} y_{0}\right) \\
& =\int_{0}^{T}\left[\frac{1}{2}\left|\bar{r} \dot{y}_{0}(t)\right|^{2}-V\left(\bar{r} y_{0}(t)\right)\right] d t \\
& =\int_{0}^{T}\left[\frac{1}{2 k^{2}}\left|\bar{r} \dot{x}_{0}\left(\frac{t}{k}\right)\right|^{2}-V\left(\bar{r} x_{0}\left(\frac{t}{k}\right)\right)\right] d t \\
& =\int_{0}^{T}\left[\frac{1}{2 k^{2}}\left|\bar{r} \dot{x}_{0}(\tau)\right|^{2}-V\left(\bar{r} x_{0}(\tau)\right)\right] d \tau \\
& <\int_{0}^{T}\left[\frac{1}{2}\left|\bar{r} \dot{x}_{0}(\tau)\right|^{2}-V\left(\bar{r} x_{0}(\tau)\right)\right] d \tau \\
& =\varphi\left(\bar{r} x_{0}\right) \leq \varphi\left(x_{0}\right)=\inf _{x \in \mathscr{M}} \varphi(x),
\end{aligned}
$$

which is a contradiction. Hence $x_{0}$ has minimal period $T$.

Proof of Corollary 2. For $x \in \mathbb{R}^{N}$ with $|x|=1$, set $a(\lambda)=$ $\lambda^{-1}\left(V^{\prime}(\lambda x), x\right)$. Then (V8) implies that

$$
\begin{aligned}
a^{\prime}(\lambda)=\frac{1}{\lambda^{3}} & {\left[\left(V^{\prime \prime}(\lambda x) \lambda x, \lambda x\right)\right.} \\
& \left.-\left(V^{\prime}(\lambda x), \lambda x\right)\right]>0 ;
\end{aligned}
$$

that is, $a(\lambda)$ is increasing strictly on $(0,+\infty)$. Thus (V4) is satisfied. It is easy to check that $V$ satisfies (V1)-(V3) when (V8) is available.

Without assumption (V5), $\varphi$ may not be continuous differentiable on $H$. However, we can use the method introduced in [1] to handle such a situation. Let $K>1$ and $\chi \in$ $C^{\infty}\left(\mathbb{R}^{+}, \mathbb{R}^{+}\right)$such that $\chi(s)=1$ if $s \leq K, \chi(s)=0$ if $s \geq K+1$, and $\chi^{\prime}(s)<0$ if $s \in(K, K+1)$. Set

$$
V_{K}(x)=\chi(|x|) V(x)+[1-\chi(|x|)] \rho(K)|x|^{4},
$$

where $\rho(K) \geq \max _{K \leq|x| \leq K+1}\left(V(x) /|x|^{4}\right)$. Since $V$ satisfies (V8), it follows from Lemma 2.3 in [18] that

$$
\begin{array}{r}
0<(\nu+1) V(x) \leq\left(V^{\prime}(x), x\right), \\
\forall x \in \mathbb{R}^{N} \backslash\{0\} .
\end{array}
$$

Arguing similarly to Lemma 2.9 in [1], we have

$$
0<\lambda V_{K}(x) \leq\left(V_{K}^{\prime}(x), x\right), \quad \forall x \in \mathbb{R}^{N} \backslash\{0\},
$$

where $\lambda=\min \{v+1,4\}>2$. Let $a_{3}=\min _{|x|=1} V_{k}(x)$ and $a_{4}=\max _{|x|=1} V_{k}(x)$. Since $K>1$, then $a_{3}=\min _{|x|=1} V(x)>0$ and $a_{4}=\max _{|x|=1} V(x)>0$. Obviously, $a_{3}, a_{4}$ are independent of $K$. Arguing similarly to Lemma 4.1 of [22], we can prove the following inequality:

$$
V_{K}(x) \geq a_{3}|x|^{\lambda}-a_{4}, \quad \forall x \in \mathbb{R}^{N} .
$$

Hence $V_{K}$ satisfies (V1)-(V6).

Consider the disturbed second order Hamiltonian systems

$$
\ddot{x}+V_{K}^{\prime}(x)=0,
$$

whose variational function is

$$
\varphi_{K}(x)=\int_{0}^{T}\left[\frac{1}{2}|\dot{x}(t)|^{2}-V_{K}(x(t))\right] d t .
$$

Since $V_{K}$ satisfies (V1)-(V6), applying Theorem 1, (38) possesses a periodic solution $x_{K}$ with minimal period $T$.

Claim. There exists $K_{0}>0$ independent of $K$ such that $\left\|x_{K}\right\|_{L^{\infty}}<K_{0}$.

Let $x_{1}(t)=b_{1} \sqrt{T /\left(2 \pi^{2}\right)} \sin (2 \pi t / T) \in E$ with $b_{1}=$ $(1,0, \ldots, 0)^{\prime} \in \mathbb{R}^{N}$, where $\left(^{\prime}\right)$ denotes the transposition of a vector. Computing directly, we have

$$
\left\|x_{1}\right\|^{2}=\left\|x_{1}\right\|_{1}^{2}=1, \quad\left\|x_{1}\right\|_{L^{2}}^{2}=\frac{T^{2}}{4 \pi^{2}} .
$$

So $x_{1} \in S$. It follows from (37) that

$$
\begin{aligned}
\varphi_{K}\left(r x_{1}\right) & =\frac{1}{2} r^{2}-\int_{0}^{T} V_{K}\left(r x_{1}\right) d t \\
& \leq \frac{1}{2} r^{2}-a_{3} r^{\lambda}\left\|x_{1}\right\|_{L_{\lambda}}^{\lambda}+a_{4} T \\
& \leq \frac{1}{2} r^{2}-a_{3} M_{1}^{\lambda} r^{\lambda}\left\|x_{1}\right\|_{L^{2}}^{\lambda}+a_{4} T \\
& \leq \frac{1}{2} r^{2}-a_{3} M_{1}^{\lambda} r^{\lambda} \frac{T^{\lambda}}{(2 \pi)^{\lambda}}+a_{4} T=h(r),
\end{aligned}
$$

where $M_{1}$ denotes the constant such that $\|x\|_{L^{\lambda}} \geq M_{1}\|x\|_{L^{2}}$. Since $\lambda>2, h(r) \rightarrow-\infty$ as $r \rightarrow+\infty$. Then there exists $M_{2}>0$ independent of $K$ such that $h(r) \leq M_{2}$. Thus

$$
\begin{aligned}
c_{K} & =\inf _{x \in M} \varphi_{K}(x)=\inf _{x \in S} \max _{r \in \mathbb{R}^{*}} \varphi_{K}(r x) \\
& \leq \max _{r \in \mathbb{R}^{*}} \varphi_{K}\left(r x_{1}\right) \leq \max _{r \in \mathbb{R}^{*}} h(r) \leq M_{2} .
\end{aligned}
$$


Hence

$$
\begin{aligned}
M_{2} & \geq c_{K}=\varphi_{K}\left(x_{K}\right) \\
& =\int_{0}^{T}\left[\frac{1}{2}\left(V_{K}^{\prime}\left(x_{K}\right), x_{K}\right)-V_{K}\left(x_{K}\right)\right] d t \\
& \geq \frac{\lambda-2}{2} \int_{0}^{T} V_{K}\left(x_{K}\right) d t .
\end{aligned}
$$

Consequently, we have

$$
\begin{aligned}
c_{\infty}^{-2}\|x\|_{L^{\infty}}^{2} \leq\|x\|^{2}= & 2 \varphi_{K}\left(x_{K}\right) \\
& +2 \int_{0}^{T} V_{K}\left(x_{K}\right) d t \leq \frac{2 \lambda}{\lambda-2} M_{2} .
\end{aligned}
$$

It follows that there exists $K_{0}>0$ independent of $K$ such that $\left\|x_{K}\right\|_{L^{\infty}} \leq K_{0}$. If $K>K_{0}$, by the definition of $V_{K}, V_{K}=V$ and hence $x_{K}$ is a nonconstant $T$-periodic solution of (1) with $A=0$.

Proof of Theorem 3. Since $V$ is even, so do $\varphi$ and $\Psi$. Since $\inf _{x \in S} \Psi(x)=c_{0}>0$ and $\Psi$ satisfies the (PS) condition, then Lemma 6 yields that $\Psi$ has infinitely many pairs of critical points. According to Lemma 13, $\varphi$ has infinitely many pairs of critical points. Thus (1) has infinitely many pairs of periodic solutions.

Example 15. Let $A=0$ and let $V$ be

$$
V(x)=|x|^{2}\left[\ln \left(1+|x|^{2}\right)\right]^{2} .
$$

It is easy to check that $V$ does not satisfy (V8) or even (V7) (cf. [23]). However, $V$ satisfies (V1)-(V6). Applying Theorems 1 and 3 , for any $T>0$, (1) possesses infinitely many pairs of $T$-periodic solutions and has at least one solution with $T$ as its minimal period.

\section{Conflict of Interests}

The author declares that there is no conflict of interests regarding the publication of this paper.

\section{Acknowledgments}

This project is supported by NSFC (no. 11301102) and by PCSIRT (no. IRT1226).

\section{References}

[1] P. H. Rabinowitz, "Periodic solutions of Hamiltonian systems," Communications on Pure and Applied Mathematics, vol. 31, no. 2, pp. 157-184, 1978.

[2] Y. M. Long, "The minimal period problem of classical Hamiltonian systems with even potentials," Annales de l'Institut Henri Poincaré. Analyse Non Linéaire, vol. 10, pp. 605-626, 1993.

[3] Y. M. Long, "The minimal period problem of periodic solutions for autonomous superquadratic second order Hamiltonian systems," Journal of Differential Equations, vol. 111, no. 1, pp. 147174, 1994.
[4] Y. M. Long, "On the minimal period for periodic solutions of nonlinear Hamiltonian systems," Chinese Annals of Mathematics B, vol. 18, no. 4, pp. 481-484, 1997.

[5] G. Fei, S. Kim, and T. Wang, "Solutions of minimal period for even classical Hamiltonian systems," Nonlinear Analysis. Theory, Methods \& Applications, vol. 43, pp. 363-375, 2001.

[6] G. Fei and T. Wang, "The minimal period problem for nonconvex even second order Hamiltonian systems," Journal of Mathematical Analysis and Applications, vol. 215, no. 2, pp. 543559, 1997.

[7] G. Fei and T. Wang, "Some results on the minimal period problem of nonconvex second order Hamiltonian systems," Chinese Annals of Mathematics B, vol. 20, no. 1, pp. 83-92, 1999.

[8] Z. Nehari, "On a class of nonlinear second-order differential equations," Transactions of the American Mathematical Society, vol. 95, pp. 101-123, 1960.

[9] Z. Nehari, "Characteristic values associated with a class of nonlinear second-order differential equations," Acta Mathematica, vol. 105, pp. 141-175, 1961.

[10] A. Pankov, "Periodic nonlinear Schrödinger equation with application to photonic crystals," Milan Journal of Mathematics, vol. 73, pp. 259-287, 2005.

[11] A. Pankov, "On decay of solutions to nonlinear Schrödinger equations," Proceedings of the American Mathematical Society, vol. 136, no. 7, pp. 2565-2570, 2008.

[12] A. Szulkin and T. Weth, "Ground state solutions for some indefinite variational problems," Journal of Functional Analysis, vol. 257, no. 12, pp. 3802-3822, 2009.

[13] A. Szulkin and T. Weth, "The method of Nehari manifold," in Handbook of Nonconvex Analysis and Applications, D. Y. Gao and D. Motreanu, Eds., pp. 597-632, International Press, Boston, Mass, USA, 2010.

[14] M. Willem, Minimax Theorems, vol. 24 of Progress in Nonlinear Differential Equations and Their Applications, Birkhäuser, Boston, Mass, USA, 1996.

[15] G. Chen and S. Ma, "Periodic solutions for Hamiltonian systems without Ambrosetti-Rabinowitz condition and spectrum 0," Journal of Mathematical Analysis and Applications, vol. 379, no. 2, pp. 842-851, 2011.

[16] Y. W. Ye and C. L. Tang, "Periodic solutions for second order Hamiltonian systems with general superquadratic potential," Bulletin of the Belgian Mathematical Society. Simon Stevin, vol. 21, no. 1, pp. 1-18, 2014.

[17] A. Ambrosetti and G. Mancini, "Solutions of minimal period for a class of convex Hamiltonian systems," Mathematische Annalen, vol. 255, no. 3, pp. 405-421, 1981.

[18] Y. M. Xiao, "Periodic solutions with prescribed minimal period for the second order Hamiltonian systems with even potentials," Acta Mathematica Sinica, vol. 26, no. 5, pp. 825-830, 2010.

[19] S. T. Deng, "Minimal periodic solutions of a class of Hamiltonian systems," Acta Mathematica Sinica, vol. 27, no. 5, pp. 664675, 1984 (Chinese).

[20] P. Magrone, Critical point methods for indefinite nonlinear Elliptic equations and Hamiltonian systems [Ph.D. thesis], Universit a Degli Studi Di Roma “Tor Vergata”, 2001.

[21] Q. Wang, Z. Wang, and J. Shi, "Subharmonic oscillations with prescribed minimal period for a class of Hamiltonian systems," Nonlinear Analysis. Theory, Methods \& Applications, vol. 28, no. 7, pp. 1273-1282, 1997. 
[22] J. Mawhin and M. Willem, Critical Point Theory and Hamiltonian Systems, Springer, New York, NY, USA, 1989.

[23] G. Fei, "On periodic solutions of superquadratic Hamiltonian systems," Electronic Journal of Differential Equations, no. 2002, pp. 1-12, 2002. 


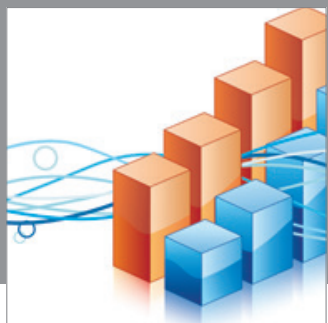

Advances in

Operations Research

mansans

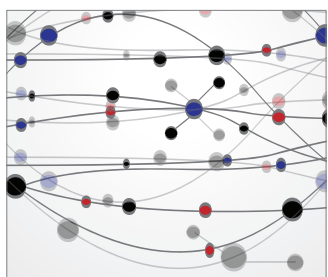

The Scientific World Journal
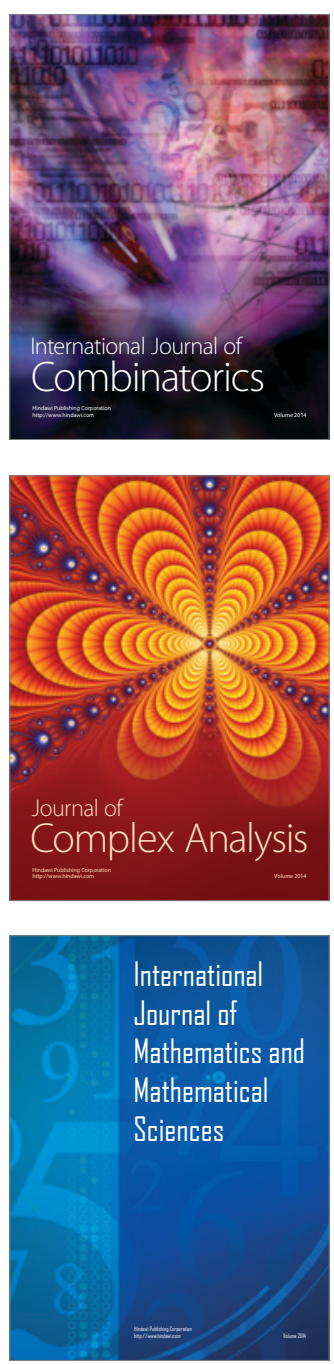
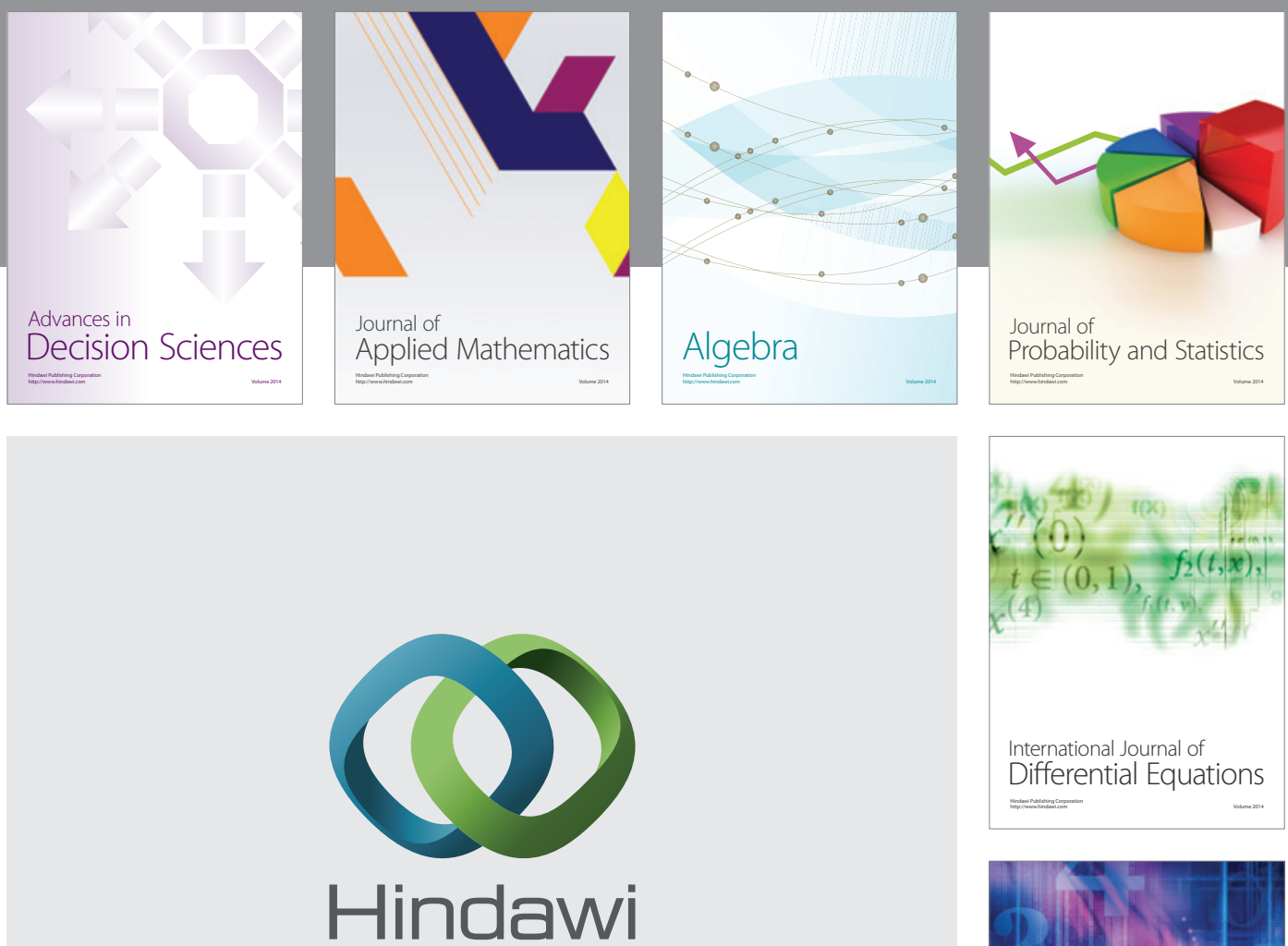

Submit your manuscripts at http://www.hindawi.com
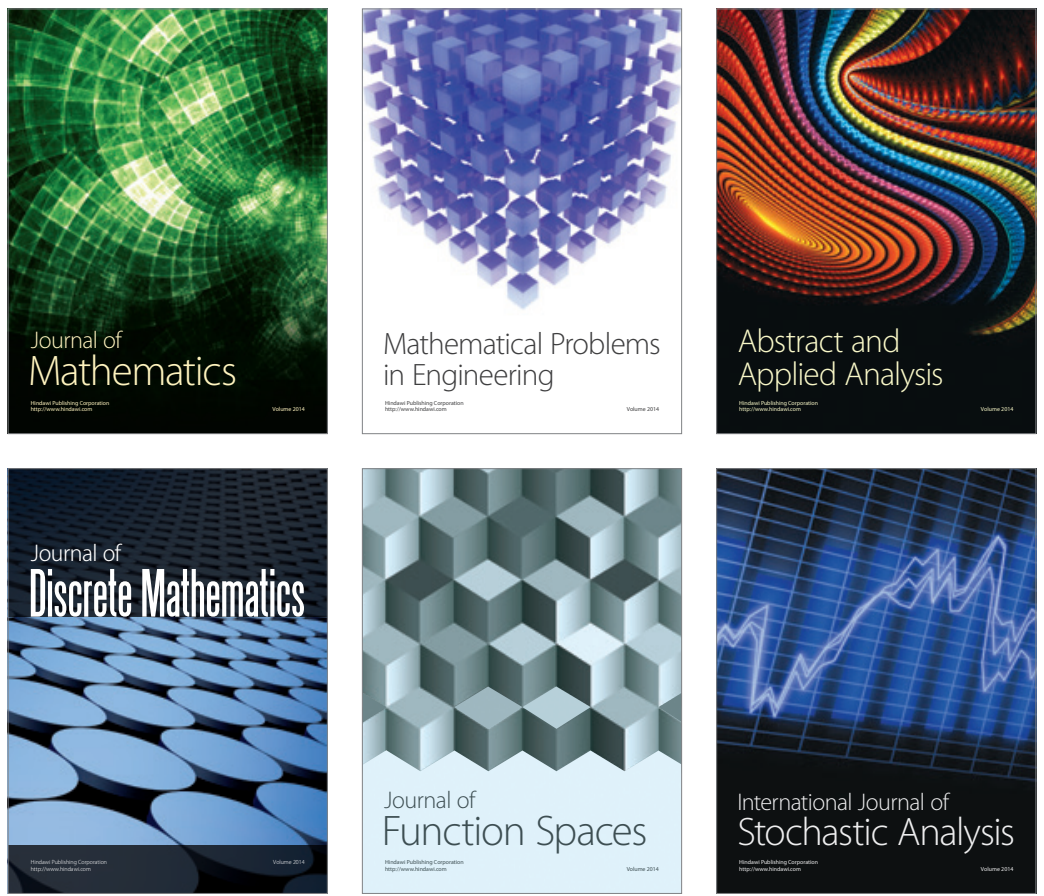

Journal of

Function Spaces

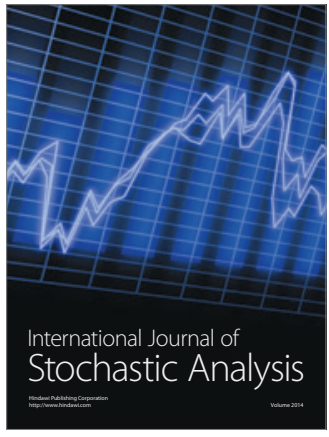

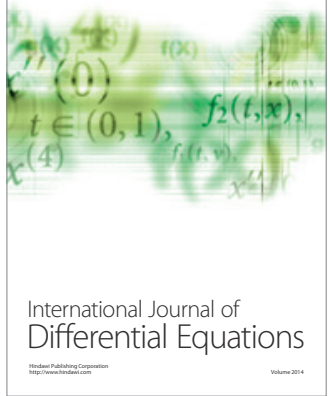
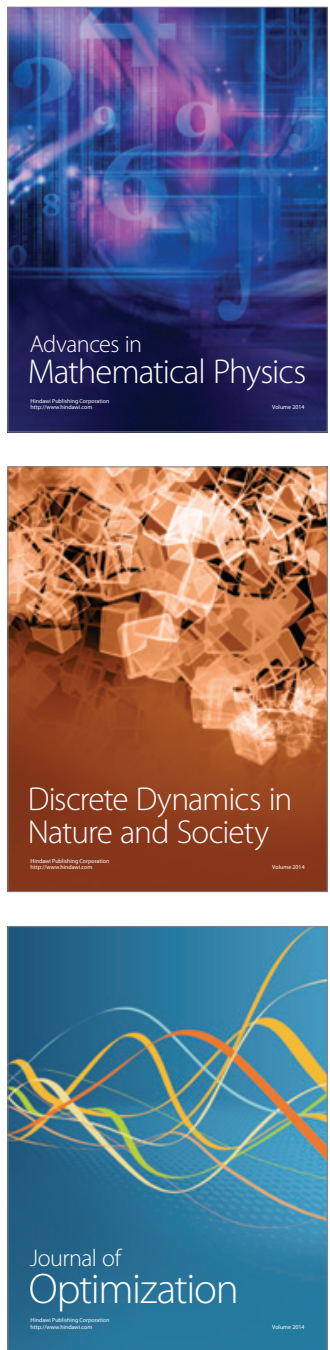